Europe. These earthworms were obtained either by experienced 'pickers', each collecting up to 10,000 Lumbricus terrestris per night from golf courses, or by breeding Eisenia foetida or Eudrilus eugeniae in a wide range of human or organic wastes. The potential of earthworms in degradation of organic wastes and as a source of food and drugs was reviewed by J. Sabine (Waite Institute, Adelaide, Australia) and discussed by contributors from several other countries as far afield as the US, Japan, Nigeria and the Phillipines. Most of these groups have concentrated on the production of a material suitable as a plant growth medium or a soil additive, from a wide range of organic wastes, by the action of earthworms.

There seems little doubt that Darwin's faith in the usefulness of earthworms to man has been fully justified and it seems likely that these humble creatures may prove to be even more valuable during the next decade.

\title{
The biology of oligodendroglia
}

from a correspondent

OLIGODENDROGI.IAL cells have the important role of producing the myelin sheath of axons in the central nervous system. The formation of myelin results in changes that facilitate the conduction of impulses. The study of oligodendroglia and myelin is therefore of considerable importance in neurobiology and has relevance to several human diseases, particularly multiple sclerosis (MS).

At a workshop* held in June, recent biochemical studies of myelin in whole brain or in tissue slices and of oligodendrocytes in mixed cell cultures were discussed. These investigations provide new insights into the development and differentiation of oligodendrocytes, the synthesis of myelin and gene expression of oligodendrocytes in some nonmyelinating systems. It appears that, during development, the expression of enzymes responsible for the synthesis of myelin components and the production of these components are coordinated but that many of these events do not occur simultaneously. For example, the activity of cerebroside sulphotransferase (CST), the enzyme that converts cerebroside to sulphatide, rises before that of UDP galactose:ceramide galactosyltransferase (CGalt), the enzyme that catalyses the last step of cerebroside synthesis. This puzzling finding may imply that most of the cerebroside formed before myelination is channeled into sulphatide formation. Both these myelin lipids can be detected before myelin basic protein (MBP) appears. Several other enzymes of lipid synthesis also increase dramatically before and during myelination and then decline. Although the control mechanisms that regulate such enzyme activity are poorly understood, some interdependencies have now been established. For example, the activity of the enzyme glycerol phosphate dehydrogenase is induced specifically in oligodendrocytes by cortisol and the

-A multidisciplinary, international group of about 60 investigators gathered in June at Arlie tlouse, Virginia, under the auspices of the National Ifultiple Sclcrosis Society of America to discuss recent advances in the biology and pathology of oligodendroglial cells. oligodendrocyte-specific enzyme 2 ' $3^{\prime}$-cyclic nucleotide $3^{\prime}$ phosphodiesterase is stimulated in glioma lines by noradrenaline via a cyclic AMP-dependent mechanism. The activity of CST appears partially to depend on the ratio of cholesterol to lecithin in membranes containing the enzyme; such ratios can be altered experimentally by adding appropriate liposomes in vitro.

It has also been possible to localize several enzymes (including CST, CGalt and plasmalogenase) to oligodendrocytes using bulk-isolated enriched cell fractions.

The assembly of myelin components by the cell into myelin membranes is being examined by kinetic studies combined with protein synthesis inhibitors. The two major myelin proteins, MBP and proteolipid protein (PLP), are assembled into myelin at different times after synthesis: the incorporation of PLP into myelin lags behind that of MBP by several minutes and continues for several minutes after protein synthesis has stopped. These data imply that PLP is processed after synthesis and is assembled into a different precursor membrane than MBP. Preliminary results indicate that it is possible to isolate populations of precursor membrane vesicles that represent both different stages in the formation of myelin precursors and separate pathways for carrying different myelin proteins.

After assembly, the myelin constituents turnover quite rapidly and at different rates. Constituents added early in development are removed at a slower rate than those added later ('last in, first out'). Each lipid has its own turnover time but, in general, lipids turnover faster than proteins and some proteins formed early in life are metabolically very stable. The more rapidly metabolized proteins may not be homogeneously distributed within the sheath but are located preferentially in areas peripheral to compacted myelin layers.

Many of these observations on the biochemical behaviour of oligodendroglia in situ have been confirmed by investigators studying oligodendroglia in mixed cell culture systems. In vitro preparations show the developmental increases of differentiated properties of oligodendrocytes, such as CST, CGalt, cerebroside and MBP. There is speculation that, whereas Schwann cells will not express differentiated functions in the absence of axons, the expression of these properties in oligodendroglia is partly preprogrammed into the cell and does not require feedback signals from axons for induction of many of its components.

Several other recent in vivo and in vitro studies provide new insights into the role of cell interactions in myelination. Addition of rat glia from dissociated embryonic spinal cord to cultures of dorsal root ganglia results in proliferation of the ensheathing cells and also in extensive myelination - additional proof that peripheral nervous system axons can promote glial mitosis and differentiation. In vivo experiments using mouse chimaeras obtained by aggregation of eight-cell preimplantation embryos have recently permitted the study of interactions between normal and mutant glia throughout primary development. Chimaeras and other techniques such as nerve grafting may also elucidate the interactions between the glial cells themselves and between glia and axons in the abortive remyelination and regeneration that follows various forms of central nervous system injury. Additional benefits will be derived from recent improvements in the preparation of separate cultures of astroglia and oligodendroglia and from the very exciting advances made in the development of specific markers for different types of glia and neurone.

Using immunological techniques, glia and neurones can be identified and purified independently of morphological characteristics. This methodology has been revolutionized by procedures for the derivation of monoclonal antibodies. Purified cell populations should help characterize the molecules involved in myelin induction and in the control of glial cell proliferation.

It is not yet known whether the oligodendrocytes or the myelin sheath itself is the main target in MS. Using organotypic central nervous system cultures and adding myelinotoxic MS sera in the presence of complement, there is a selective destruction of oligodendrocytes in vitro. However these observations have not been corroborated in MS lesions displaying ongoing demyelination where oligodendroglia do not appear to be selectively destroyed in the early lesions. Immunocytochemical studies of MS plaques also suggest that the mechanism of myelin breakdown in MS may be immuneligand-mediated phagocytosis rather than a failure of the oligodendrocytes. Thus, in MS, the mechanisms that lead to the loss of myelin-forming cells and to the limited repair of demyelination remain to be clarified. 